\title{
Functional evaluation of professional athletes treated with a mini-open technique for achilles tendon rupture
}

\author{
Antonio Vadalà \\ Riccardo Maria Lanzetti \\ Alessandro Ciompi \\ Cristina Rossi \\ Domenico Lupariello \\ Andrea Ferretti
}

UOC Ortopedia e Traumatologia, Ospedale S.Andrea, "Sapienza" University of Rome, Italy

Corresponding author:

Riccardo Maria Lanzetti

UOC Ortopedia e Traumatologia, Ospedale S.Andrea, "Sapienza" University of Rome, Italy

Via Grottarossa, 1035

00189 Roma, Italy

E-mail riccardolanzetti@gmail.com

\section{Summary}

Introduction: in this study we report the functional results of $\mathbf{3 6}$ professional athletes treated with a combined percutaneous and mini-open technique. Methods: patients were evaluated with Victorian Institute of Sports Assessment-Achilles questionnaire (VISA-A), the objective 100-points Hannover scale and the Ergo-jump Bosco System device.

Results: at a mean 28- month follow-up no re-rupture cases were observed. Six patients had minor complications. The Ergo-jump Bosco System device showed no significant differences in the side-to-side evaluation in regard to strength ($0.94 \%)$ and elasticity $(+2.44 \%)$, while a significant post-operative loss was detected in the endurance trials $(-6.78 \%)$. The Hannover scale showed an average score of 94.5 , while the VISAA had an average of 93.1. Thirty-one patients resumed their pre-operative sports activity level within five months from surgery.

Conclusions: our results showed that the combined mini-open and percutaneous repair is an effective treatment for professional athletes, with satisfactory clinical and functional results, lack of major complications and a quick return to professional sports activity.

KEY WORDS: Achilles tendon, mini-open technique, athletes, jumping capability.

\author{
Introduction
}

Achilles tendon ruptures represent one of the most common tendon injuries among athletes, especially those involved in acceleration/deceleration or jumping activities. Men in their third or fourth decade of life, with lack of prodromal symptoms, are the prototype of patients affected by this kind of rupture ${ }^{1}$. In fact, this usually occurs on tendons which are already affected by degenerative disorders, in which sport activities ${ }^{2-5}$ simply act as trigger factor6-9.

Despite a very high satisfaction rate reported with many surgical procedures, such as the open, the mini-open, or the percutaneous procedure, many major intra- and post-operative complications are still reported, lowering the overall satisfaction rate of patients and sometimes delaying their return to sports. This is even more important if we consider professional athletes involved in very demanding sport activities.

The hypothesis of this study is to verify if the miniopen technique modified by Kakiuchi ${ }^{10}$ is an effective treatment in professional athletes with subcutaneous Achilles tendon rupture.

\section{Materials and methods}

We prospectively followed-up 36 patients surgically treated for spontaneous Achilles tendon rupture at our Sports Injury Centre ${ }^{11}$. They were all operated on between 2008 and 2010 with a combined mini-open and percutaneous technique as described by Kakiuchi (1995). Inclusion criteria to enter the study were: involvement in sports activities as professional players; lack of previous diseases both in the involved and in the healthy tendon; lack of previous local injections to the involved and healthy side; surgical treatment within five days from the trauma; a minimum follow-up of 24 months. We excluded from the study patients who had undergone previous systemic therapy with corticosteroids or patients affected by collagen fiber disorders.

In all patients, the combined open and percutaneous repair was carried out by the same senior author trained in Sports Traumatology. No augmentations were ever used. In all cases, post-operative protocol consisted in two weeks immobilization in a non-weight bearing cast with 15 degrees of plantar flexion, followed by additional two weeks of immobilization in a weight-bearing walker brace in neutral position. 
After this period, all patients started a comprehensive physical rehabilitation program, basically focused on recovery of range of motion (ROM) in the first two weeks, on increasing the endurance and strength of the triceps muscle and restoring coordination and proprioception of the musculotendinous triceps unit, after the first two weeks.

\section{Surgical Technique}

All patients were surgically treated with a combined percutaneous and mini-open procedure as described by Kakiuchi in $1995^{10}$. With the patient in prone position, a $3.9 \mathrm{~cm}$ longitudinal paramedian incision was made just above the palpable tendon gap, to provide a direct view of the rupture. After longitudinal incision of the fascia and paratenon, two suture guides made of $2 \mathrm{~mm}$ Kirschner wire were inserted medially and laterally to the proximal tendon stump: a long straight needle threaded with an absorbable $2 \#$ suture was then passed transversely through the skin, the intact tendon, and the holes of the suture guides. After removal of the two Kirschner guides, the proximal tendon stump was easily drawn down by pulling on the sutures (Fig. 1). The same procedure was repeated on the distal stump of the torn tendon so that the two stumps could be easily sutured together by passing the sutures on the opposite stump and tightening.

\section{Follow-up}

Among the 36 surgically treated athletes, thirty-six were available for follow-up. There were 33 males $(91.6 \%)$ and 3 females $(8.4 \%)$. The mean age was 29.7 (range: 21-35).

In all cases, the tendon rupture occurred during sport activities: in particular, in five cases (13.8\%) during an official match and in 31 cases $(86.2 \%)$ during training. Patients' sports activities are shown in Figure 2.

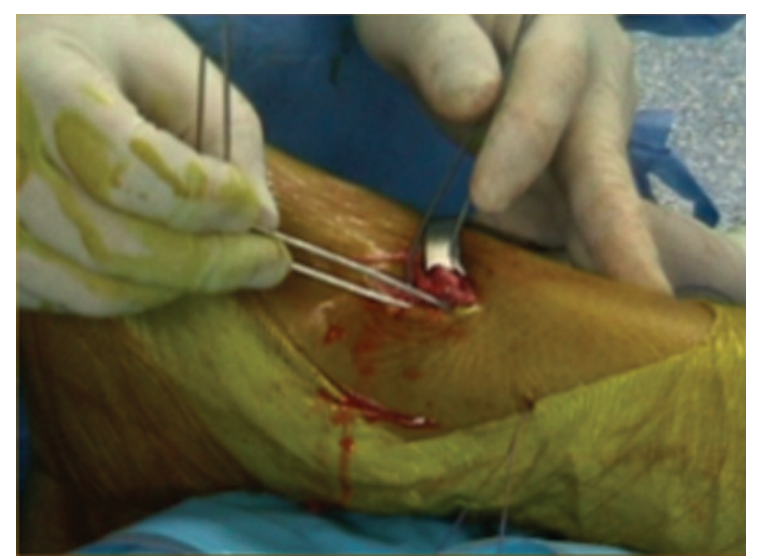

Figure 1. Bilateral insertion of two looped K-wires on the proximal stump of the achilles tendon.

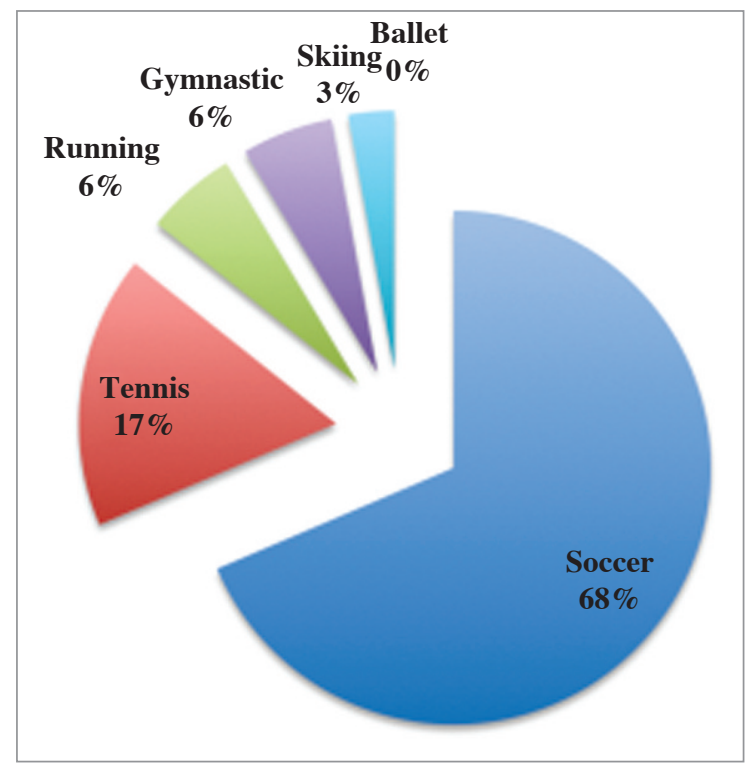

Figure 2. Patients' sports activity.

All the patients were followed-up after an average of 28 months (range: 24-31).

An independent blinded examiner assessed all the patients with an accurate physical examination and with two validated scoring scales: the Victorian Institute of Sports Assessment-Achilles questionnaire (VISA-A $)^{12}$ and the objective 100-points Hannover scale. Moreover, all the patients were functionally evaluated with the Ergo-jump Bosco System device (Byomedic, SCP, Barcelona, Spain), with the aim to objectively estimate the recovery of the musculotendinous triceps unit. Charts of the patients were examined before follow-up. All patients gave their informed consent to participate in this study. The physical examination consisted of an evaluation of the skin incision, in order to detect any hypertrophic scar tissue or skin adhesions; skin sensitivity all around the incision area was assessed as well. The calf muscle circumference was measured (side-to-side, S/S) $5 \mathrm{~cm}$ distal to the tibial tuberosity: diameter differences were graded as mild $(\leq 1 \mathrm{~cm})$, moderate $(\leq 2 \mathrm{~cm})$, and severe $(>2 \mathrm{~cm})$. Moreover, the ankle range of motion and tendon width (S/S) were also recorded.

\section{Hannover Scale}

The Hannover-100-point-scale combines in a simple scale the clinical and isokinetic evaluation of the ankle function, compared to the contralateral limb. In our study we chose this scale because it allowed an objective evaluation of the Achilles tendon function and its recovery, compared to the healthy side. The articular range was evaluated with a goniometer $\left(0^{\circ}\right.$ was set as starting position) and the calf muscle circumference was measured at the point of maximum thickness. 


\section{Functional evaluation}

The functional evaluation was made with the use of the Bosco Ergo-jump System, which allows to measure flight time and height of one or more consecutive vertical jumps in order to evaluate the triceps musculotendinous unit ${ }^{13}$.

We chose this method to obtain a reliable index of the recovery of jumping capability which needs a great contribution from the Achilles tendon.

Athletes were assessed using three different jump tests: Squatting Jump test, Counter Movement Jump test, and Ten Repetition Counter Movement Jump test. The first test is designed to determine the explosive strength of the extensor muscles of the inferior limbs: the athlete squats until the knees are flexed at 90 degrees and then jumps vertically with maximum intensity. Hands are placed on the hips and no countermovement is used. The best score of three non-consecutive single leg jumps was recorded. The Counter Movement Jump test is designed to determine the elasticity of the extensor muscles of the inferior limbs: the athlete starts in a standing position and then jumps vertically with maximum intensity. A countermovement of lower and upper limbs is used. The best score of three non-consecutive single leg jumps was recorded. The Ten Repetition Counter-movement Jump is designed to determine the muscle resistance of the inferior limbs: the athlete starts in a standing position and then jumps vertically with maximum intensity. A counter-movement of lower and upper limbs is used. Results of ten consecutive single leg jumps were recorded.

All functional evaluations were performed side-toside. The data obtained from the study was analysed using the Chi square test and the Fisher exact test; the SPSS software for Windows, version 8.0 (SPSS, Inc., Chicago, IL) was used for all analyses.

\section{Results}

At a mean follow-up of 28 months no major complications, such as re-ruptures, thrombophlebitis or pulmonary embolisms were registered. We did not record major complications in patients treated with this combined percutaneous and mini-open technique.

Six patients $(16.6 \%)$ showed minor complications, consisting in skin adhesions in two cases (5.5\%), the presence of a hypertrophic scar in one case $(2.7 \%)$ and wound healing delay with superficial infection in three patients $(8.1 \%)$, as documented by charts' records. One of the two patients with skin adhesions underwent a new surgical procedure to solve the aesthetic complication (she is a professional ballet dancer), even thought she reported no functional restrictions related to the skin adhesions. No cases of sural entrapment or sensibility disorders were observed. The ankle range of motion (S/S) was complete in 31 patients $(86.1 \%)$; a $5^{\circ}$ loss of dorsal flexion was registered in 4 patients $(11.1 \%)$. Calf atrophy was observed in twenty-eight patients $(77.7 \%)$ ( $p: 0.02)$ : calf circumference differences (S/S) were $\leq 1 \mathrm{~cm}$ in 16 patients $(44.4 \%), \leq 2 \mathrm{~cm}$ in 14 patients $(38.8 \%)$, and $>$ $2 \mathrm{~cm}$ in 6 patients (16.6\%). An increase of the repaired tendon width was detected in 30 patients $(83.3 \%)$ (p:0.02): twenty-nine patients $(80.5 \%)$ showed a thickness twice the contralateral, and 1 patient $(2.7 \%)$ a thickness more than twice the contralateral.

Evaluation scales and functional tests results are shown in Table 1, Table 2, and Table 3 respectively.

\section{Return to Sport}

Thirty-one patients (86\%) resumed their pre-operative sports activity level within five months from surgery. Out of the other five patients $(14 \%)$, two resumed their sports activity within seven months, and three between seven and ten months. This delay was due to an additional operation in one patient (skin adhesion debridement) and superficial wound infection in two patients. Thirty-three patients (91\%) reported they resumed the same pre-operative sports activity level, the last three patients were forced to decline their sport activity level (Tab. 4).

Table 1. VISA-A scale results.

\begin{tabular}{|l|c|}
\hline & VISA-A \\
\hline Excellent $(>90)$ & 24 pts. $(66.6 \%)$ \\
\hline Good $(75-90)$ & 11 pts. $(30.5 \%)$ \\
\hline Poor $(65-75)$ & 1 pt. $(2.7 \%)$ \\
\hline Unsatisfactory $(<65)$ & $/$ \\
\hline
\end{tabular}

Table 2. Hannover scale results.

\begin{tabular}{|l|c|}
\hline & HANNOVER \\
\hline Very good (>90) & 27 pts. (75\%) \\
\hline Good (80-89) & 9 pts. (25\%) \\
\hline Satisfactory (70-79) & $/$ \\
\hline Fair (60-69) & $/$ \\
\hline
\end{tabular}

Table 3. Functional evaluation tests results.

\begin{tabular}{|l|c|}
\hline & $\begin{array}{c}\text { Ergo-jump Bosco } \\
\text { system evaluation }\end{array}$ \\
\hline Squatting jump (S/S) & $-0,94 \%$ \\
\hline Counter movement (S/S) & $+2.44 \%$ \\
\hline Repetitive jump (S/S) & $-6.78 \%$ \\
\hline
\end{tabular}


Table 4. Post-operative sport level.

\begin{tabular}{lll}
\hline & $=$ Pre operative & Decline sport level \\
\hline $\begin{array}{l}\text { Post-operative } \\
\text { level }\end{array}$ & $33(91 \%)$ & $3(9 \%)$ \\
\hline
\end{tabular}

\section{Discussion}

Surgical suture of Achilles tendon rupture represents the most used treatment for athletes. In fact, many authors demonstrate a low rate of side effects after a mini invasive surgical technique. Despite this advantage, side effects affect about $15-20 \%$ of all patients operated on with different techniques: these include adhesions with surrounding soft tissues, unsuccessful recovery of the original length, appearance of surgical scar, increased re-rupture rate, inactivity time and delay of complete recovery of previous activities ${ }^{1,14-17 .}$ The main finding of this study is represented by the satisfactory clinical and functional results provided by the combined mini-open and percutaneous technique in patients involved in professional sports activities. In fact, even though it is now well accepted how surgical treatment is the gold standard for young active patients, it is matter of debate among authors which type of technique provides the best results in terms of functional recovery in high-demand athletes.

The authors' results regarding the best technique to treat athletes are still very discordant ${ }^{2,}$ 18-22. In particular, the introduction of the percutaneous repair in 1977 by Ma and Griffith ${ }^{23}$ aimed to accomplish the quarrel between supporters of the open technique and supporters of the conservative treatment: its aim was to gather the advantages of surgery, such as minor re-rupture rate, faster recovery, shorter immobilization, and the advantages of the conservative technique, such as minor vascular or neurological complications, superficial or deep infections ${ }^{7,24-28}$.

Maffulli et al. ${ }^{18}$ published the results of seventeen elite athletes surgically treated with a percutaneous technique, reporting excellent results, both in terms of time of return to sports (average of 4.8 months) and in terms of successful outcome (no re-rupture cases and return to pre-operative sport activity in all the cases). Moreover, they reported only two cases of minor complications such as superficial infection of the wound. Similarly, Gigante et al. ${ }^{19}$, in a prospective randomized study, showed how the percutaneous technique offered clinical outcomes equivalent to the open technique, with considerable lower cutaneous complications, faster recovery and better patients' compliance. However, other authors, such as Aracil et al. ${ }^{2}$ and Wong et al. ${ }^{20}$, did not report the same positive results and complained about high re-rupture rates and sural nerve injuries. Some authors tried to reinforce their surgical procedure with the use of autologous or synthetic augmentations, but even in these cases results were not uniform ${ }^{29-32}$.

The new combined open and percutaneous technique shown by Kakiuchi (1995) represented a further at- tempt to conjugate the benefits coming from the open and the percutaneous technique. Rebeccato et al. ${ }^{33}$ compared three groups of patients operated on with open, percutaneous and combined mini-open and percutaneous technique, and showed how this last group reported a re-rupture rate comparable to open procedure, but with significant $(p<0.01)$ less calf atrophy, more strength of the involved limb and better ankle range of motion.

Results obtained in our study are in line with what shown by Kakiuchi (1995) and Rebeccato et al. (2001) since neither re-ruptures nor other major complications were detected. Moreover, patients included in this study were all professional athletes, with a high request for a satisfactory recovery of the pre-traumatic activity level. For this reason, the mini open technique could be considered a valid choice for professional athletes with Achilles tendon rupture. In fact, in line with the literature ${ }^{34}$, in our experience all patients recovered their sport activities: 33 patients (91\%) returned to their pre-operative sport level and only 3 patients were forced to decline their level.

The combined open and percutaneous technique appeared to satisfy the request of the patients, with both subjective and objective satisfactory clinical outcomes (VISA-A: 93.1; Hannover: 94.5) and functional results: in particular, the Ergo-Jump tests performed showed very satisfactory recovery of the triceps strength, as well as the elasticity of the operated limb ( $-0.94 \%$ and $+2.44 \%$ respectively; $p$ : n.s.).

The only significant deficit detected was in regard to the endurance of the triceps unit (evaluated through the Counter Movement Jump test) which showed an average deficit of $6.78 \%$; however, this functional results did not correlate with clinical results, since the vast majority of the patients returned to their pre-traumatic sports activity level.

We decided to use the Bosco System and the Hannover scale for our study because we believe they can provide a clinical and functional evaluation of the whole musculotendinous unit.

A limitation of this study is certainly represented by the lack of a control group; however, all objective measurements and functional evaluations were assessed in a side-to-side way, thus allowing us to have a intrinsic control group. For the same reason, we did not compare results of the used surgical technique with another surgical technique.

In conclusion, our results showed that the combined mini-open and percutaneous repair is an effective and reliable surgical treatment for professional athletes with satisfactory clinical and functional results, lack of major complications and fast return to professional sports activity.

\section{References}

1. Wong J, Barrass V, Maffulli N. Quantitative review of operative and nonoperative management of Achilles tendon ruptures. Am J Sports Med. 2002;30(4): 565-575.

2. Aracil J, Pina A, Lozano JA, Torro V, Escriba I. Percutaneous 
suture of Achilles tendon ruptures. Foot Ankle. 1992;13(6): 350-351.

3. Inglis $A E$, Sculco TR. Surgical repair of ruptures of the tendon Achilles. Clin Orthop. 1981;156:160-169.

4. Inglis AE, Scott WN, Sculco TR, Patterson AH. Ruptures of the tendo achillis: an objective assessment of surgical and non surgical treatment. J Bone Joint Surg. 1976;58A:990-993.

5. Kongsgaard M, Aagaard P, Kjaer M, Magnusson SP. Structural Achilles tendon properties in athletes subjected to different exercise modes and in Achilles tendon rupture patients. J Appl Physiol. 2005;99(5):1965-1971.

6. Arner O, Lindholm Å, Orell SR. Histologic changes in subcutaneous rupture of the Achilles tendon. A study of 74 cases. Acta Chir Scandinavica. 1958;116:484-490.

7. Jozsa L, Kvist M, Balint BJ, Reffy A, Jarvinen M, Lehto M, Barzo M. The role of recreational sport activity in Achilles tendon rupture. A clinical, pathoanatomical, and sociological study of 292 cases. Am J Sports Med. 1989;17:338-343.

8. Jozsa L, Lehto M, Kvist M, Balint JB, Reffy A. Alterations in dry mass content of collagen fibers in degenerative tendinopathy and tendon-rupture. Matrix. 1989;9:140-146.

9. Kannus P, Jozsa L. Histopathological changes preceding spontaneous rupture of a tendon. A controlled study of $891 \mathrm{pa}-$ tients. J Bone Joint Surg Am. 1991;73:1507-1525.

10. Kakiuchi M. A combined open and percutaneous technique for repair of tendon Achillis. J Boint Joint Surg. 1995;77B:6164.

11. Padulo J, Oliva F, Frizziero A, Maffulli N. Muscles, Ligaments and Tendons Journal. Basic principles and recommendations in clinical and field science research. MLTJ. 2013;4:250-252.

12. Robinson JM, Cook JL, Purdam C, et al. Victorian Institute Of Sport Tendon Study Group. The VISA-A questionnaire: a valid and reliable index of the clinical severity of Achilles tendinopathy. Br J Sports Med. 2001;35(5):335-341.

13. De Carli $A$, Vadalà $A$, Ciardini $R$, lorio R, Ferretti A. Spontaneous Achilles tendon ruptures treated with a mini-open technique: clinical and functional evaluation. J Sports Med Phys Fitness. 2009;49(3):292-296.

14. Khan RJ, Carey Smith RL. Surgical interventions for treating acute Achilles tendon ruptures. Cochrane Database Syst Rev. 2010;(9): CD003674.

15. Jiang N, Wang B, Chen A, Dong F, Yu B. Operative versus nonoperative treatment for acute Achilles tendon rupture: a meta-analysis based on current evidence. Int Orthop. 2012;36(4):765-773.

16. Wilkins R, Bisson LJ. Operative versus nonoperative management of acute Achilles tendon ruptures: a quantitative systematic review of randomized controlled trials. Am J Sports Med. 2012;40(9):2154-2160.

17. Willits K, Amendola A, Bryant D, Mohtadi NG, Giffin JR, Fowler P, Kean CO, Kirkley A. Operative versus Nonoperative Treatment of Acute Achilles Tendon Ruptures. A Multicenter Randomized Trial Using Accelerated Functional Rehabilitation. J Bone Joint Surg Am. 2010; 92:2767-2775.

18. Maffulli N, Longo UG, Maffulli GD, Khanna A, Denaro V.
Achilles tendon ruptures in elite athletes. Foot Ankle Int 2011;32(1):9-15.

19. Gigante A, Meschini A, Verdenelli A, Del Torto M, Ulisse S, De Palma L. Open versus percutaneous repair in the treatment of acute Achilles tendon rupture: a randomized prospective study. Knee Surg Sports Traumatol Arthros. 2008;16(2):204-209.

20. Wong J, Barrass V, Maffulli N. Quantitative review of operative e nonoperative management of achilles tendon ruptures. Am J Sports Med. 2002; 30(4):565-575

21. Jiang N, Wang B, Chen A, Dong F, Yu B. Operative versus nonoperative treatment for acute Achilles tendon rupture: a meta-analysis based on current evidence. Int Orthop. 2012;36(4):765-773.

22. Al-Fakayh O. Outcome following mini-invasive surgical repair of closed Achilles tendon rupture. Int Orthop. 2010;34(1):149.

23. Ma GV, Griffith TG. Percutaneous repair of acute closed ruptured achilles tendon: a new technique. Clinic Orthop Relat Res. 1977;(128):247-255.

24. Bradley JP, Tibone JE. Percutaneous and open surgical repairs of Achilles tendon ruptures. A comparative study. Am J Sports Med. 1990;18:188-195.

25. Kangas J, Pajala A, Ohtonen P, Leppilahti J. Achilles tendon elongation after rupture repair: a randomized comparison of 2 postoperative regimens. Am J Sports Med. 2007;35(1):59-64. Epub 2006.

26. Rippstein P, Easley M. "Mini-open" repair for acute Achilles tendon ruptures. Tech Foot Ankle Surg. 2006;5(1):3-8.

27. Hockenbury RT, Johns JC. A biomechanical in vitro comparison of open versus percutaneous repair of tendon Achilles. Foot Ankle. 1990;11(2):67-72.

28. Thompson J, Baravarian B. Acute and chronic Achilles tendon ruptures in athletes. Clin Podiatr Med Surg. 2011;28(1):117135. Review.

29. Haas F, Seibert FJ, Koch H, Hubmer M, Moshammer HE, Pierer G, Scharnagl E. Reconstruction of combined defect of the Achilles tendon and the overlying soft tissue with e fascia lata graft and a free fasciocutaneous lateral arm flap. Ann Plast Surg. 2003;51:376-383.

30. Basiglini L, lorio R, Vadalà A, Conteduca F, Ferretti A. Achilles tendon surgical revision with synthetic augmentation. Knee Surg. Sports Traumatol Arthrosc. 2010;18(5):644-647.

31. Schneppendahl J, Thelen S, Schek A, et al. Initial stability of two different adhesives compared to suture repair for acute Achilles tendon rupture-A biomechanical evaluation. Int Orthop. 2012;36(3):627-632.

32. Wegrzyn J, Luciani JF, Philippot R, Brunet-Guedj E, Moyen B, Besse JL. Chronic Achilles tendon rupture reconstruction using a modified flexor hallucis longus transfer. Int Orthop. 2010;34(8):1187-1192. Epub 2009.

33. Rebeccato A, Santini S, Salmaso G, Nogarin L. Repair of the achilles tendon rupture: a functional comparison of three surgical techniques. J Foot Ankle Surg. 2001;40(4):188-194.

34. Guillo S, Del Buono A, Dias M, Denaro V, Maffulli N. Percutaneous repair of acute ruptures of the tendo Achillis. Surgeon. 2013;11(1):14-19. 